\title{
PREDICCIÓN DEL ABANDONO UNIVERSITARIO: VARIABLES EXPLICATIVAS Y MEDIDAS DE PREVENCIÓN
}

\author{
PREDICTION OF UNIVERSITY DROP-OUT: EXPLANATORY VARIABLES \\ AND PREVENTIVE MEASURES
}

\author{
Ana Belén Bernardo Gutiérrez \\ Rebeca Cerezo Menéndez \\ José Carlos Núñez Pérez \\ Ellian Tuero Herrero \\ María Esteban García \\ Universidad de Oviedo
}

Fecha de recepción: 01/03/2015

Fecha de aceptación: 09/06/2015

\section{RESUMEN}

El abandono de los estudios universitarios es un problema cuyos costes son altos tanto para el individuo como para la sociedad. Es por ello que la prevención del mismo es fundamental y cobra especial relevancia en el actual contexto de crisis económica.

Diversos autores han desarrollado investigaciones con el objetivo de establecer modelos predictivos de este fenómeno (Castaño, Gallón, Gómez y Vásquez, 2004; Trevizán, Beltrán y Cosolito, 2009; Goldenhersh, Coria y Saino, 2011; Sánchez, 2014). En este artículo se analizan dichos trabajos, identificando las ventajas y desventajas de las metodologías más utilizadas; análisis correlacionales, análisis de regresión logística, análisis de supervivencia y minería de datos.

La investigación cuyos resultados aquí se exponen, aplica la primera de las metodologías mencionadas, a fin de comprobar -en lo que respecta al fenómeno del abandono- el valor predictivo de las variables rendimiento académico previo, fecha de matriculación, rendimiento en primer curso de universidad y asistencia a clase.

Los resultados confirman la relación de dichas variables con el fenómeno estudiado. Dichos resultados son consistentes con los obtenidos por diversos autores a lo largo del tiempo, y en base a ellos se proponen dos tipos de medidas; por un lado, acciones encaminadas a facilitar el diagnóstico respecto al problema del abandono, y por otro lado, medidas encaminadas a su prevención.

Palabras clave: Universidad, educación superior, abandono, cambio de titulación, permanencia, orientación académica, orientación profesional

\begin{abstract}
University drop-out is a problem whose costs are high for both the individual and society. That is the reason why prevention is essential and it is particularly important in the current economic crisis context.

Several authors have conducted research in order to establish predictive models of this phenomenon (Castaño, Gallon, Gómez and Vásquez, 2004; Trevizán, Beltrán and Cosolito, 2009; Goldenhersh, Coria and Saino, 2011; Sánchez, 2014). This article analyses
\end{abstract}


these works, identifying the advantages and disadvantages of the most used methods; correlational analysis, logistic regression, survival analysis and data mining.

The research whose results are presented here applies the first of the mentioned methodologies, with the aim to test -in regard to the phenomenon of abandonmentthe predictive value of the following variables: prior academic performance, date of enrolment, performance in first year college and attendance.

The results confirm the relationship of these variables with the studied phenomenon. These results are consistent with those obtained by several authors throughout time. To finish, two types of measures based on this results are proposed; on one hand, measures to facilitate diagnosis of drop-out, on the other hand, measures aiming it prevention.

Key words: University; higher education; drop-out; change of degree; persistence; academic advising; career counselling

\section{INTRODUCCIÓN}

El abandono de los estudios universitarios es un fenómeno de alcance mundial, razón por la cual merece la atención de la comunidad científica. Es fundamental conocer bien las causas de cara a instaurar medidas preventivas que resulten eficaces, sin embargo el coste que -en ocasiones- traen aparejadas este tipo de investigaciones supone una importante limitación.

Son muchos los estudios desarrollados sobre esta temática, tanto a nivel nacional como internacional. De entre ellos destacan aquellos cuyo objetivo es establecer un modelo predictivo del abandono universitario, y al cual se pretende contribuir con este estudio.

Cabe aquí reflexionar sobre la necesidad y conveniencia de llegar a tales extremos, ya que como Salmon (2005) explica, el concepto de predicción en Ciencias Sociales es cuestionado por diversos autores, debido a la naturaleza del objeto de estudio de éstas en contraposición con aquellos de las ciencias experimentales.

Así pues el debate intensificado desde mediados del siglo XX sobre la predictibilidad del comportamiento humano y las predicciones en el campo de las Ciencias Sociales, continúa hasta nuestros días. Sin embargo, las predicciones -sean sobre acontecimientos futuros o sobre hechos ya acaecidos que han pasado inadvertidos- son legítimas, puesto que se sustentan en conocimientos validados por métodos aceptados por la ciencia. No obstante, es importante tener presente que dichas predicciones están vinculadas a un contexto concreto, quedando su validez afectada por el cambio de diversas circunstancias. 
Una vez expuesta la necesidad de utilizar las predicciones realizadas de manera cautelosa, se comentan las cuatro principales metodologías aplicadas al estudio del abandono con el fin de realizar predicciones:

En primer lugar es necesario mencionar los estudios basados en el análisis de relaciones entre variables, cuyo objetivo es identificar las asociaciones más importantes entre el fenómeno estudiado y las variables influyentes, así como la interacción entre éstas en su caso. Este tipo de estudios gozan de determinadas ventajas entre las que cabe destacar la identificación de variables concretas con una importante influencia en el fenómeno, estando algunas de ellas basadas en datos secundarios disponibles en los sistemas informáticos de toda universidad. La principal desventaja de estos estudios es la limitación explicativa del fenómeno estudiado, quedando determinado porcentaje de abandono sin explicar por diversos motivos (por ejemplo por el surgimiento de nuevas situaciones, cambios en la situación laboral o familiar, etc.) o con una difícil interpretabilidad de las correlaciones en términos del significado intrínseco de las variables.

Ejemplo de este tipo de estudios es el desarrollado por Araque, Roldán y Salguero (2009). Los autores, tras compilar la información disponible en las bases de datos de la Universidad de Granada de una amplia muestra de estudiantes procedentes de 25 titulaciones diferentes y de realizar los pertinentes análisis correlacionales, identifican aquellas variables con influencia relevante en el abandono para cada facultad participante, así como aquellas comunes a las tres facultades participantes (edad de ingreso, nivel educativo de los padres y vía de acceso a la universidad).

Por su parte Marín, Infante y Troyano (2000) realizan una investigación sobre el fracaso académico en la universidad que, si bien no cuenta con una muestra amplia ( $\mathrm{N}=103$ desertores de la Universidad de Sevilla), sí resulta interesante por la cantidad de información recogida y analizada, dado que además de los clásicos cuestionarios y entrevistas personales, aplican test estandarizados de inteligencia, motivación e intereses y preferencias profesionales. Los autores concluyen que "los aspectos motivacionales y actitudinales poseen un potencial predictor del fracaso académico igual o mayor que los aspectos cognoscitivos o intelectuales" (Marín et al., 2000:506).

Por último, dentro de las investigaciones con carácter predictivo basadas en análisis correlacionales, es relevante la investigación desarrollada por Willcoxson (2010) sobre la intención de abandono en la facultad de negocios de la Universidad de Melbourne. La misma 
identifica las variables que se asocian con esta intención en cada uno de los semestres de la titulación, sentando las bases para el establecimiento de medidas preventivas específicas según el momento en que se encuentra el estudiante. De entre sus resultados, cabe destacar una mayor serie de variables implicadas en la intención de abandono en primer curso de carrera en comparación con el resto de cursos y, dentro de éstas, el importante rol desempeñado por las interacciones personales.

En segundo lugar, se encuentran los estudios cuyo objetivo es el establecimiento de un modelo predictivo del abandono basados en el análisis de la regresión logística simple o múltiple. Este tipo de investigaciones, tras identificar aquellas variables que correlacionan con el abandono de titulación, construyen ecuaciones de regresión que posibilitan la realización de predicciones en base a los datos en ellas utilizados. La principal ventaja que presentan es la selección de las variables que mayor peso ejercen en el fenómeno, así como el conocimiento de estos pesos para la cohorte estudiada, posibilitando la aplicación de medidas preventivas a discreción. Sin embargo, la capacidad predictiva de la fórmula obtenida puede verse limitada a la cohorte estudiada si se dan cambios en las variables contempladas, precisando realizar de nuevo la recogida y análisis de datos para modificar o reajustar la ecuación tras el cambio de circunstancias.

Ejemplo de este tipo de análisis es el realizado por Trevizán et al. (2009) que, utilizando los datos de 1276 ingresantes en la facultad de ciencias agrarias de la universidad Nacional de Rosario entre los años 1994 y 2000. Los autores obtienen un modelo de regresión logística relativo al abandono de los estudios en dicha facultad basado principalmente en cuatro variables: Edad de ingreso, escolaridad del padre, escolaridad de la madre y tipo de residencia.

Por su parte, en el contexto español Rodrigo, Molina, García-Ros y Pérez-González (2012) analizan la capacidad predictiva de determinadas variables sociodemográficas y educativas en el abandono de la titulación de Psicología por parte de dos cohortes de estudiantes de nuevo ingreso. El análisis, basado en la comparación de modelos de regresión logística anidados, da lugar a un modelo refinado en el que las variables de mayor peso son: sexo, modalidad de bachillerato, dedicación (a tiempo completo o parcial), grado de preferencia de los estudios matriculados en la preinscripción, residencia familiar y nivel de estudios de los padres.

Un enfoque metodológico novedoso por ser reciente su aplicación al estudio de la deserción escolar es el de los análisis de 
supervivencia y los modelos de duración que de estos análisis se desprenden. Esta metodología de investigación, ampliamente utilizada en el campo de la medicina, consistente en observar un conjunto de sujetos en el tiempo hasta el momento en que se produce el suceso e identificar los patrones de mortandad (en este caso, abandono). Este tipo de metodología permite recoger las particularidades del fenómeno estudiado en su dimensión longitudinal, sin embargo esta misma característica puede resultar una desventaja, al traer aparejado un gran volumen de trabajo que implica mayor necesidad de recursos humanos y económicos. En este sentido, es interesante la experiencia de Castaño et al. (2004), que tras analizar la cohorte de nuevo ingreso en 1996 en la facultad de ingeniería de la Universidad de Antioquia, bajo modelos de riesgo proporcional obtienen evidencia de la importancia conjunta de factores individuales, académicos, socioeconómicos e institucionales en la explicación y predicción del abandono de titulación.

Por último, se localizan estudios que aplican técnicas novedosas en su aplicación en el campo de la Educación, como son diferentes métodos basados en la inteligencia artificial. Como principal ventaja destaca su alta capacidad predictiva sobre el alumnado de la cohorte estudiada, sin embargo esta capacidad se ve limitada para las sucesivas cohortes de nuevo ingreso, puesto que tanto el contexto como la propia institución de acogida suelen sufrir cambios de un año para otro, lo que implica la necesidad de una nueva recogida de datos y un nuevo ajuste del modelo.

De entre los escasos ejemplos de aplicación de la minería de datos al estudio del abandono universitario, cabe destacar el trabajo de Sánchez. El mismo consiste en encontrar un modelo capaz de predecir el abandono en el primer año de carrera en el Grado de Ingeniería Informática. Para ello, el autor analiza los datos de tres cohortes de nuevo ingreso (2009 - 2011) a partir de los datos disponibles en el sistema informático de la universidad, diseñando y probando diversos modelos y seleccionando el mejor de ellos (aquel que aplica el algoritmo Random Forest). Según declara Sánchez (2014:69), el modelo seleccionado tiene una probabilidad de acierto de aproximadamente el $80 \%$, minimizando el número de casos de alumnos clasificados como persistentes que finalmente optan por abandonar.

Las diversas opciones de estudio anteriormente analizadas perseguían la descripción y predicción del abandono universitario, sin embargo es necesario recordar el debate sobre la predicción aplicada al campo de las Ciencias Sociales, ya que aunque ésta es posible, es recomendable asumir una postura cautelosa en su interpretación. No en 
vano autores como Goldenhersh et al. (2011) tras lograr un modelo predictivo con una capacidad de acierto del $95 \%$ de los abandonos, han visto como esta probabilidad de acierto disminuye paulatinamente para cada cohorte de nuevo ingreso, descendiendo hasta el $77 \%$ transcurridos cinco años.

Una vez realizado un recorrido por los principales trabajos que tienen por objetivo la predicción del abandono de los estudios en educación superior, el presente artículo se plantea el objetivo de ampliar el conocimiento sobre esta cuestión centrándose en la cohorte de nuevo ingreso en el curso 2010/11. Se ha seleccionado esta cohorte por ser la primera en la que la Universidad de Oviedo ofertaba todas sus titulaciones ya adaptadas al Espacio Europeo de Educación Superior (EEES), lo que supone ampliar el conocimiento a disposición de la comunidad científica en este nuevo contexto, dada la escasez de estudios realizados sobre el mismo.

El objetivo de la investigación que aquí se presenta es la identificación de variables con mayor influencia en el abandono de titulación para la corte de nuevo ingreso en la Universidad de Oviedo durante el curso 2010/11.

Las variables influyentes finalmente seleccionadas responden a dos criterios:

1. Disponibilidad: Se han seleccionado aquellas variables que habitualmente están a disposición de la comunidad universitaria, bien por estar registradas en los sistemas informáticos de la institución (nota de acceso, fecha de matriculación y rendimiento en primer curso), bien por estar su registro al alcance de los docentes (asistencia a clase), sin la necesidad de aplicar instrumento alguno para la recogida de datos, abaratando con ello los costes de investigación.

2. Consistencia: Complementariamente, se ha comprobado la coincidencia de los resultados obtenidos con los publicados por otros autores, lo que informa de su consistencia a lo largo del tiempo y en diversos tipos de Instituciones de Educación Superior (Araque et al., 2009 y Casaravilla, Del Campo, García y Torralba el al., 2012, entre otros)

Es menester puntualizar que esta metodología permite la selección de alumnado para la aplicación de actuaciones preventivas sin costes añadidos, ya que minimiza el coste de los estudios exploratorios que las han de preceder, lo que supone una gran ventaja 
para la institución universitaria ante el actual contexto de crisis económica (Smink y Schargel, 2013).

\section{MÉTODO.}

\section{Descripción de los participantes.}

Se ha estudiado la cohorte de nuevo ingreso en la Universidad de Oviedo en el curso 2010/11. Esta población ascendía a 5215 estudiantes de los que, en septiembre de 2012, 4194 permanecían en la titulación inicialmente matriculada, 363 habían cambiado de titulación a otra dentro de la Universidad y 658 habían abandonado la Universidad de Oviedo.

Tras aplicar un procedimiento de muestreo aleatorio estratificado, tomando como estratos cada una de las ramas de conocimiento, se ha seleccionado una muestra final de 1055 participantes, formada por dos submuestras con un nivel de confianza del $97 \%$ y con un margen de error del 4\%: permanecen en la titulación inicialmente matriculada $(\mathrm{N}=626)$ y abandonan ésta $(\mathrm{N}=429)$, estableciendo cuotas para la submuestra de abandono de titulación, en función de si el participante se había matriculado en otra titulación de la Universidad de Oviedo (cambio de titulación) o no (abandono).

Así, la distribución de la muestra se corresponde con la propia de la población y, por tanto, es representativa de ésta, lo que hace posible la generalización de resultados. La tabla 1 sintetiza la distribución final de la muestra empleada en la investigación.

\begin{tabular}{|l|c|c|c|}
\cline { 2 - 4 } \multicolumn{1}{c|}{} & Permanece & Cambia & Abandona \\
\hline Arte y humanidades & $61,9=\mathbf{6 2}$ & $13,83=\mathbf{1 4}$ & $42,75=\mathbf{4 3}$ \\
\hline Ciencias & $\mathbf{5 2 , 3 = \mathbf { 5 2 }}$ & $11,74=\mathbf{1 2}$ & $21,38=\mathbf{2 1}$ \\
\hline Cc. Salud & $82,56=\mathbf{8 3}$ & $6,7=7$ & $17,17=\mathbf{1 7}$ \\
\hline Cc. Soc y jurídicas & $258,09=\mathbf{2 5 8}$ & $69,17=\mathbf{6 9}$ & $110,67=\mathbf{1 1 1}$ \\
\hline $\begin{array}{l}\text { Ingeniería y } \\
\text { Arquitectura }\end{array}$ & $171,05=\mathbf{1 7 1}$ & $\mathbf{5 0 , 7 2 = \mathbf { 5 1 }}$ & $83,84=\mathbf{8 4}$ \\
\hline TOTAL & $\begin{array}{c}\mathbf{6 2 6} \\
\text { participantes }\end{array}$ & $\begin{array}{c}\mathbf{1 5 3} \\
\text { paticipantes }\end{array}$ & $\begin{array}{c}\mathbf{2 7 6} \\
\text { participantes }\end{array}$ \\
\hline
\end{tabular}

Tabla $n^{\circ} 1$ : Distribución de los alumnos que han conformado la muestra en función de su situación académica transcurridos dos cursos académicos tras su ingreso en la institución. 


\section{Variables e Instrumentos.}

En un primer momento se ha extraído información disponible acerca de la población estudiada en la aplicación informática de gestión académica de la universidad. En concreto se han extraído tanto la información de contacto precisa para la realización de la entrevista, como determinados datos sociodemográficos (nacionalidad, tamaño familiar, nivel de estudios y ocupación de los padres) como otras variables relativas a su ingreso y progreso en la universidad (rendimiento académico previo, fecha de matriculación, titulación matriculada, área correspondiente y número de créditos matriculados, presentados y aprobados).

En un segundo momento se aplicó mediante llamada telefónica o email un cuestionario diseñado ad hoc para el estudio. Los ítems del cuestionario hacían referencia a:

- Estado civil, nivel de renta y tipo de residencia durante el curso.

- Motivos de elección tanto de la Universidad de Oviedo como de la titulación matriculada en el curso 2010/11.

- Acciones de acogida universitaria en las que ha participado y valoración del grado de acogida recibido.

- Dedicación al estudio, al trabajo doméstico y a un trabajo remunerado.

- Valoración del esfuerzo requerido por la titulación y satisfacción con las calificaciones.

- Valoración de las relaciones establecidas tanto con los docentes de su titulación como con sus pares.

- Planteamiento de la posibilidad de abandonar la titulación y motivos, en su caso.

- En caso de interrupción o abandono de los estudios, situación actual y grado de satisfacción con dicho cambio.

- Satisfacción general con la Universidad de Oviedo (grado en que la recomendarían).

\section{Procedimiento.}

Se ha aplicado un diseño de investigación ex-post-facto bajo un enfoque holístico, siendo éste el más adecuado dadas las características de la institución y el carácter cultural y contextual del problema estudiado.

Como se ha mencionado anteriormente, el procedimiento de recogida de información se ha llevado a cabo en dos fases o momentos: 
- Solicitud de datos personales y expediente académico de los alumnos al servicio de matriculación de la universidad

- Aplicación de un cuestionario diseñado ad hoc, llevado a cabo vía email o telefónicamente.

Los análisis descriptivos y correlacionales sobre la información recogida se han realizado mediante el programa $R$ Development Core Team (versión 2.15, disponible en http://www.R-project.org).

\section{RESULTADOS}

A continuación se exponen los resultados relativos al análisis correlacional de las variables independientes nota de acceso, fecha de matriculación, asistencia a clase y rendimiento en primer curso para con la variable dependiente grupo. La variable grupo clasifica a los participantes en el estudio según su situación académica transcurridos dos cursos desde su ingreso en la institución, a saber; permanece en la titulación inicialmente matriculada, cambia de titulación o abandona la Universidad de Oviedo.

En primer lugar, se ha explorado la relación entre el grupo al que pertenecen los alumnos (variable grupo) y la nota de acceso a la universidad, constatándose esta relación al encontrar diferencias estadísticamente significativas pero solamente entre los grupos abandona y permanece ( $p$-valor=0.019) (y no encontrándose diferencias estadísticamente significativas entre el grupo Cambia y Permanece $p$-valor $=0.2$ ), siendo la media de 8.18 para el grupo Abandona y 1.45 puntos más alta para el grupo Permanece.

Considerando que incorporarse a los estudios universitarios una vez iniciadas las clases puede jugar en contra del rendimiento del estudiante y puede contribuir a plantearse la posibilidad de abandonar, se ha examinado la relación entre las variables "grupo" y "fecha de matriculación", dándose una tendencia para los grupos de Abandono y Cambio de Titulación una tendencia a matricularse una vez iniciadas las clases y la tendencia inversa para el grupo de Permanecia ( $p$-valor=0.000), tal y como se puede observar en el gráfico 1. 


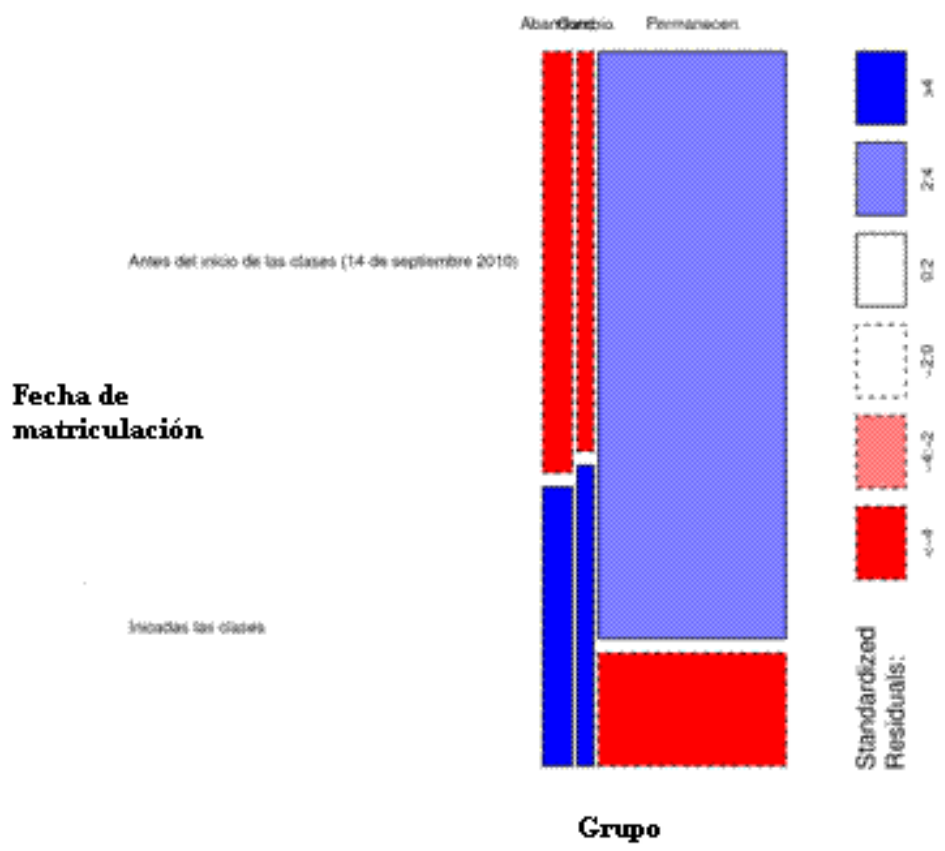

Gráfico $n^{\circ}$ 1: Relación entre la variable grupo y la fecha de primera matriculación en la universidad.

Considerando que la asistencia a clase facilita el aprendizaje al tiempo que contribuye a una mejor integración social y académica en la universidad, se ha examinado la relación entre las variables "grupo" y "grado de asistencia a clase" encontrando relación entre ellas ( $p$ valor=0.000):

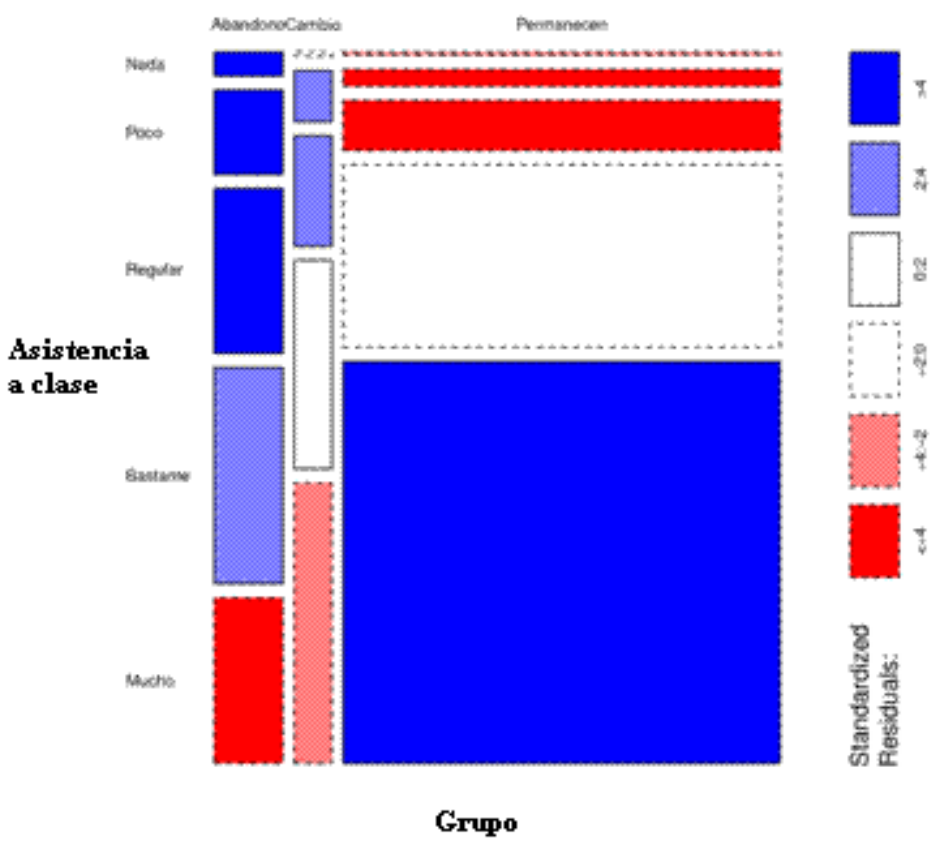

Gráfico $n^{\circ} 2$. Relación entre la variable grupo y el grado de asistencia a clase declarado por los participantes. 
De la observación del gráfico 2 se deduce que el grado de asistencia a clase es menor a la de una distribución gaussiana para el grupo de Abandono y Cambio, sin embargo se da una tendencia inversa en el grupo de Permanencia, lo cual indica que los alumnos que asisten bastante 0 mucho a clase tienen mayores probabilidades de Permanecer que los que asisten nada, poco o regular.

En cuanto al rendimiento académico en la universidad, se ha examinado la relación entre el número de créditos superados en el primer curso de grado y el grupo al que pertenece el alumno, encontrando diferencias estadísticamente significativas. Así, la media de créditos aprobados es de 10 créditos para los alumnos que abandonan ( $p$-valor=1.130409 e-28), 18 créditos para los que cambian de titulación ( $p$-valor=8.026733 e-06) y 44 para los que permanecen ( $p$ valor $=1.206618$ e-138).

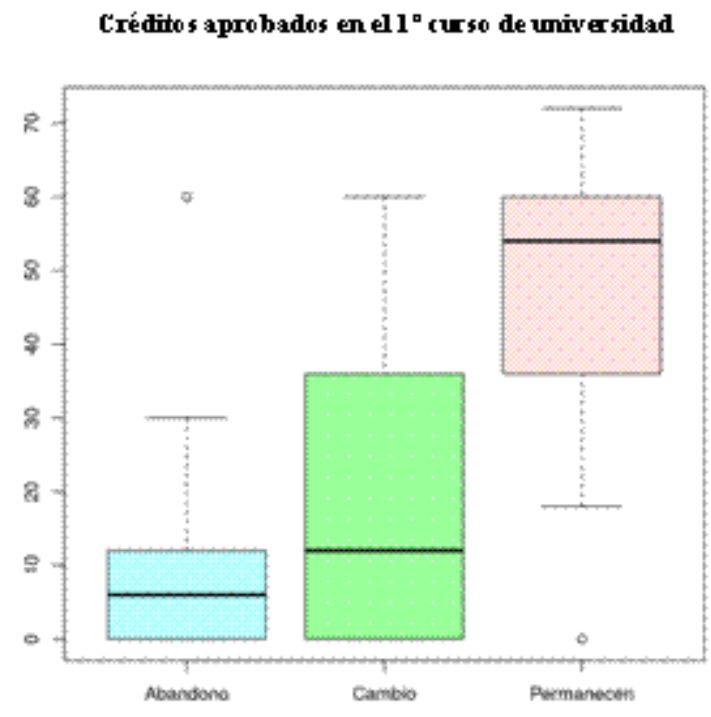

Gráfico n 3: Relación entre la variable grupo y el número de créditos aprobados en el primer curso de universidad

En el gráfico 3 se reflejan las grandes diferencias de rendimiento académico existentes entre los grupos estudiados: la puntuación más baja del grupo de alumnos que permanece es superior al percentil 75 del grupo que abandonó, así como a la media del grupo que cambia de titulación. La media de créditos superados por el grupo que permanece es superior a prácticamente todos los casos del grupo que abandona y se aproxima al número máximo de créditos superados por el grupo de cambio de titulación. 


\section{DISCUSIÓN Y CONCLUSIONES}

El objetivo de la investigación cuyos resultados aquí se han presentado era la identificación de variables influyentes en el fenómeno estudiado en base a dos criterios; Por un lado, la disponibilidad de la información sin la necesidad de aplicar instrumentos específicos para su recogida y, por otro lado, la consistencia con los resultados obtenidos por otros autores a lo largo del tiempo.

Esta metodología facilita la selección de aquellos grupos de alumnos que presentan cualidades que invitan a clasificarlos como en situación de riesgo de abandono, para la posterior aplicación discrecional de medidas preventivas y correctivas. A continuación se discuten estos resultados, contrastándolos con los obtenidos por otros investigadores, para terminar proponiendo una serie de medidas preventivas frente al abandono de titulación.

En primer lugar, se evidencia la relación entre rendimiento académico previo y abandono de titulación, en términos de nota de acceso a la universidad. Los autores del presente trabajo también han constatado esta relación en términos de nota media de bachillerato/ciclo formativo (Esteban, Bernardo, Rodríguez, Núñez y Fernández, 2013). Son muchos los investigadores que han confirmado esta relación (Smith y Naylor, 2001; Montmarquette, Mahseredjian y Houle, 2001; Rodríguez, 2004; Di Pietro, 2006; Araque et al, 2009; Belloc, Maruotti y Petrella, 2010; Saldaña y Barriga, 2010; Rodrigo et al, 2012; Casaravilla et al., 2012). Broc (2011), por su parte, afirma que esta variable es la que mayor correlación presenta con el rendimiento académico en la universidad. Así pues, es posible afirmar que un insuficiente rendimiento académico previo al ingreso en la institución universitaria aumenta el riesgo de abandono por parte de los nuevos estudiantes, razón por la cual se hace recomendable realizar un seguimiento de aquellos alumnos cuyas calificaciones -tanto en la etapa previa al ingreso como en la prueba de acceso- sean bajas.

Matricularse en la universidad una vez iniciado el periodo lectivo sitúa a los estudiantes en una posición de desventaja frente al resto de la cohorte, pues además de no haberse beneficiado de las acciones de acogida en la universidad y en la propia titulación matriculada, supone la necesidad de acceder en solitario a aquellos contenidos abordados en clase de forma previa a su ingreso. Es por ello que se ha explorado la relación de esta variable con el abandono de titulación, constatándose ésta. Araque et al (2009) y Rodrigo et al. (2012) obtienen similares resultados. Así, se hace recomendable el establecimiento de medidas 
compensatorias destinadas a aquellos alumnos cuya fecha de inserción en la titulación sea posterior al inicio del curso académico.

Se confirma también la relación entre el fenómeno del abandono y el grado de asistencia a clase, teniendo mayores probabilidades de permanecer aquellos alumnos que asisten con mayor frecuencia a ésta, como así también confirman los resultados obtenidos por autores como Braxton, Millem y Shaw (2000), García, Alvarado y Jiménez (2000), Cabrera, Bethencourt, González y Álvarez (2006) e Iñigo, Ochoa de Eribe y Otxoa-Berrio (2011). Por su parte, Casaravilla et al. (2012) califican esta variable como predictora del abandono de titulación.

En relación con lo anterior Rodríguez y Herrera (2009) y Santos y Vallerado (2013) constatan la relación de esta variable con el rendimiento académico en la universidad. Razón por la cual se hace necesario fomentar la asistencia regular a clase y la participación activa en la misma, no solo de cara a prevenir el abandono de titulación sino también a mejorar el desenvolvimiento académico del alumnado. Esta variable cobra especial importancia en el nuevo contexto del Espacio Europeo de Educación Superior y en su sistema de créditos, que demanda mayor trabajo autónomo por parte del alumnado, donde las orientaciones docentes resultan fundamentales.

Además, la asistencia a clase favorece el establecimiento de relaciones entre los discentes con sus pares y con sus docentes, lo que según Tinto (1975) ejerce de factor amortiguador de otras variables influyentes en el fenómeno del abandono, por cuanto se facilita el apoyo al alumno. Es por ello que se recomienda una potenciación de la función tutorial en la docencia universitaria, relativa tanto a la tutoría de asignatura como a la tutoría de carrera.

Por último, se ha explorado la relación entre rendimiento académico en el primer curso de universidad y el abandono, constatándose ésta. Estos resultados coinciden con los obtenidos por Montmarquette et al. (2001) y Castaño et al. (2004) que afirman que el rendimiento académico en el primer semestre de universidad juega un papel crucial en la decisión de continuar estudiando la titulación inicialmente matriculada y Casaravilla et al. (2012) que incluso la consideran como predictora del abandono. Esta variable no sólo afecta a la decisión de permanecer o abandonar la titulación, sino que tal y como De Miguel (2001) y Goldenhersh et al. (2011) afirman, el rendimiento en primer curso es un buen predictor del posterior desempeño académico del alumno a lo largo de sus estudios universitarios. 
Una vez estudiado el problema del abandono de titulación en la institución de acogida e identificadas aquellas variables con capacidad predictiva sobre éste, es momento de proponer medidas preventivas que minimicen el riesgo de que los estudiantes de nuevo ingreso opten por desertar, como así sugiere que se haga Vázquez (2013). A continuación se centrarán las propuestas en dos líneas; por un lado, la mejora de los sistemas de registro de la información sobre el alumnado y la accesibilidad a estos datos por parte del profesorado, y por otro, la potenciación de las funciones orientadoras y tutoriales.

Respecto a la primera línea, mejora de los sistemas de registro de la información y accesibilidad de los mismos, es necesario observar que gracias al desarrollo tecnológico y abaratamiento de dispositivos de almacenamiento de la información acaecido en la última década, se ha favorecido que tanto organismos públicos como privados aumenten ingentemente la cantidad de información registrada informáticamente sobre sus clientes (Sánchez, 2014). Sin embargo, de cara a la utilización de esta información con fines investigadores se dan principalmente dos problemas:

- La información en ocasiones no es debidamente cumplimentada, pudiendo quedar campos en blanco que dificultan la posterior representatividad de los resultados obtenidos tras su análisis. Es por ello que se hace preciso establecer la obligatoriedad de complementar dicha información.

- La información almacenada, generalmente, no es accesible a la comunidad académica y científica sin autorización pertinente y consiguiente tiempo de espera. Por este motivo, los autores del presente trabajo consideran indispensable la creación de nuevas aplicaciones informáticas que mejoren la accesibilidad y usabilidad de dicha información sin que ello suponga la vulneración del derecho a la protección de datos de carácter personal previsto en el Real Decreto 1720/2007, de 21 de diciembre de desarrollo de la Ley Orgánica de Protección de Datos. En este sentido, es ilustrativa la Aplicación para el Seguimiento Institucional del Abandono en el primer año (ASIA) que la Universidad Politécnica de Madrid está desarrollando (García, Blanco, Casaravilla, Castejón, Gonzalo, Mahilla, y Malinga, 2013), que demuestra la viabilidad de este tipo de propuestas. Gracias a estas herramientas, la comunidad universitaria podría realizar diagnósticos específicos para cada titulación y el seguimiento de las cohortes, favoreciendo así la mejora continua de las tasas de abandono, cambio y permanencia. 
En cuanto a la segunda medida propuesta, potenciación de las funciones orientadoras y tutoriales, los autores remiten al lector a considerar las propuestas realizadas por Martínez (2009) y complementadas por ellos mismos. En función del momento en que se encuentre el estudiante, sus necesidades de orientación y tutorización también serán diferentes:

- De forma previa al ingreso en la universidad: Los futuros universitarios precisarán orientación para el diseño de su proyecto vital, prestando especial atención a la elección vocacional y exploración de itinerarios formativos como parte del mismo, pero sin limitarse a ello, en la línea de lo planteado por Esteban y Bernardo (2014).

- En el momento del ingreso y durante los primeros meses: Dentro de este momento se engloban las acciones destinadas a la acogida del alumno, así como a su familiarización con el programa de estudio y requisitos del mismo. Es también el momento para el fomento de los hábitos de asistencia a clase y estudio así como entrenamiento en aquellas técnicas de estudio especialmente adecuadas para la titulación cursada.

- Durante el desarrollo de los estudios, los discentes precisarán ayuda para desarrollar sus competencias académicas y profesionales, así como conocer los diversos perfiles profesionales y salidas laborales que tienen a su alcance.

- En el momento de la finalización de los estudios, los egresados precisarán orientación para el establecimiento de itinerarios postgraduación, así como para mejorar las competencias relativas a su empleabilidad.

En relación con lo anterior, es necesario recordar que la orientación no puede ser prescriptiva para la toma de decisiones por parte del orientado, ya que supondría suplantar sus propias decisiones y obstaculizaría la elaboración del proyecto personal de vida, que ha de ser autoconstruido desde la autonomía y la madurez de criterio (Santana y Feliciano, 2009:326).

Por último, procede afirmar que la combinación de ambas estrategias ha probado ser eficaz en la mejora de las tasas de permanencia en la universidad. Ejemplo de ello es la experiencia desarrollada por Tiffin University, que tras el análisis de datos de una cohorte determinada en base al modelo predictivo construido a tal efecto, selecciona a aquellos estudiantes que presentan un alto riesgo de abandono y les asigna un mentor, logrando una mejora de la tasa de permanencia del 12\% (Hanover research, 2011:16). 


\section{AGRADECIMIENTOS}

El equipo de investigación agradece la financiación por parte del Ministerio de Educación, Cultura y Deporte del PRoyecto Integral de ORientación Académico-Profesional de la Universidad de Oviedo (PRIOR), que ha hecho posible el desarrollo de la investigación cuyos resultados aquí se presentan.

\section{REFERENCIAS BIBLIOGRÁFICAS}

Araque, F., Roldán, C. y Salguero, A. (2009). Factors influencing university drop out rates. Computers \& Education, 53, 563-574.

DOI: http://dx.doi.org/10.1016/i.compedu.2009.03.013

Arriaga, J., Burillo, V., Carpeño, A., y Casaravilla, A. (2011). Caracterización de los tipos de abandono. Dividamos el problema y venceremos más fácilmente. I Conferencia Latinoamericana sobre el Abandono en la Enseñanza Superior. Recuperado el 13 de julio de 2012, de http://www.clabes2011alfaguia.org.pa/ponencias/ST_1_Abandono/7_JArriaga_Perfiles\%2 Oabandono.pdf

Belloc, F., Maruotti, A. y Petrella, L. (2010). How individual characteristics affect university students drop-out: a semiparametric mixed-effects model for an Italian case study. Journal of Applied Statistics, $00(0)$, $1-15$.

Braxton, J.M., Milem, J.M. y Shaw, A. (2000). The influence of Active Learning on the College Student Departure Process: Toward a Revision of Tinto's Theory. The Journal of Higher Education, 71 (5), 569-590. DOI: http://dx.doi.org/10.2307/2649260

Broc, M.A. (2011). Voluntad para estudiar, regulación del esfuerzo, gestión eficaz del tiempo y rendimiento académico en alumnos universitarios. Revista de Investigación Educativa, 29 (1), 171-185.

Cabrera, L., Bethencourt, J. T., González, M. y Álvarez, P. (2006). Un estudio transversal retrospectivo sobre la prolongación y abandono de estudios universitarios. Revista Electrónica de Investigación y Evaluación Educativa, 12(1), 105-127.

Casaravilla, A., Del Campo, J. M., García, A. y Torralba, M. R. (2012). Análisis del abandono en estudios de ingeniería y arquitectura en la Universidad Politécnica de Madrid. II Conferencia Latinoamericana sobre el Abandono en Educación Superior. 8-9 Noviembre. Porto Alegre, Brasil.

Castaño, E., Gallón, S., Gómez, K. y Vásquez, J. (2004). Deserción estudiantil universitaria: una aplicación de modelos de duración. Lecturas de economía, 60, 39-66. 
Esteban, M. y Bernardo, A. B. (2014). e-orientación: una metodología de orientación ubicua basada en la autorregulación del aprendizaje. Ramiro, M.T. y Ramiro-Sánchez, T. (Comp.) Libro de Actas del XI Foro Internacional sobre la Evaluación de la Calidad de la Investigación y de la Educación Superior.

Esteban, M., Bernardo, A. B., Rodríguez, L. J., Núñez J. C. y Fernández, E. (2013). La influencia de la etapa previa al ingreso en la Universidad en el progreso académico del alumno universitario. Centro de Investigação em Educação (Ed.) Atas do XII Congresso Internacional Galego-Português de Psicopedagogía. Braga: Universidade do Minho.

De Miguel, M (2001). Evaluación del rendimiento en la enseñanza superior. Resultados entre alumnos procedentes de la LOGSE y del COU. Madrid: Ministerio de Educación, Ciencia y Deporte.

Di Pietro, G. (2006). Regional Labour Market Conditions and University Dropout Rates: Evidence from Italy. Regional Studies, 40(6), 617630. DOI: http://dx.doi.org/10.1080/00343400600868770

García, A., Blanco, J., Casaravilla, A., Castejón, A., Gonzalo, A., Mahilla, M. A. y Malinga, M. (2013). Protocolo de calidad para la tasa de permanencia a un año en la Universidad Politécnica de Madrid. III Conferencia Latinoamericana sobre el abandono en educación superior. 13-15 de noviembre de 2013, Méjico.

García, M. V., Alvarado, J. M. y Jiménez, A. (2000). La predicción del rendimiento académico: regresión lineal versus regresión logística. Psicothema, 12(2), 246-252.

Goldenhersh, H., Coria, A. y Saino, M. (2011). Deserción estudiantil: Desafíos de la Universidad pública en un horizonte de inclusión. Revista Argentina de Educación Superior, 3 (3), 96-120.

Hanover research (2011). Predicting College Student Retention. Disponible a 10 de febrero de 2015, en http://www.algonquincollege.com/studentsuccess/files/2014/12/Predicting-College-Student-RetentionLiterature-Review-1.pdf

Marín, M., Infante, E. y Troyano, Y. (2000). El fracaso académico en la universidad: aspectos motivacionales e intereses profesionales. Revista Latinoamericana de Psicología, 32 (3), 505-517.

Martínez, M. (2009). La orientación y la tutorial en la Universidad en el marco del espacio europeo de educación superior. Revista Fuentes, 9, 78-97.

Montmarquette, C., Mahseredjian, S. y Houle, R. (2001). The determinants of university dropouts: a bivariate probability model with sample selection. Economics of Education Review, 20, 475484. DOI: http://dx.doi.org/10.1016/S0272-7757(00)00029-7

Iñigo, X., Ochoa de Eribe, I. y Otxoa-Berrio, L. M. (2011). Análisis y estudio del abandono de los estudiantes universitarios. VIII Jornadas 
Internacionales de Innovación Universitaria. Madrid, 11 y 12 de julio de 2011.

Real Decreto 1720/2007, de 21 de diciembre, por el que se aprueba el Reglamento de desarrollo de la Ley Orgánica 15/1999, de 13 de diciembre, de protección de datos de carácter personal. Boletín Oficial del Estado, 19 de enero de 2008, 17, 4103-4136.

Rodrigo, M. F., Molina, J. G., García-Ros, R. y Pérez-González, F. (2012). Efectos de interacción en la predicción del abandono en los estudios de Psicología. Anales de Psicología, 28 (1), 113-119.

Rodríguez, J. (2004). Tasas de éxito y fracaso académico universitario: Identificación y análisis de variables psicoeducativas. Disponible a 18 de julio de 2012, en www.orienta.org.mx/docencia/Docs/Sesion-7-8/Rodríguez.pdf

Rodríguez, C. y Herrera, L. (2009). Análisis correlacional-predictivo de la influencia de la asistencia a clase en el rendimiento académico universitario. Estudio de caso en una asignatura. Profesorado: Revista de curriculum y formación del profesorado, 13(2), 1-13.

Sánchez, J. (2014). Modelos predictivos para el estudio del abandono en centros universitarios. (Trabajo Fin de Grado, Universidad Politécnica de Madrid). Disponible a 2 de febrero de 2015 en http://oa.upm.es/31205/1/PFC_JESUS_SANCHEZ_SANTAMARIA.pdf

Saldaña, M. y Barriga, O. A. (2010). Adaptación de un modelo de deserción universitaria de Tinto a la Universidad Católica de la Santísima Concepción, Chile. Revista de ciencias sociales, 16 (4), 616-628.

Salmon, M. L. (2005). Predicción en las ciencias sociales. Enrahonar, 37, 169-179.

Santana, L.E. y Feliciano, L. (2009). Dificultades en el proceso de toma de decisiones académico-profesionales: el reto de repensar la orientación en Bachillerato. Revista de Educación, 350, 323-350.

Santos, $M^{a}$ V. y Vallerado, E. (2013). Algunas dimensiones relacionadas con el rendimiento académico de estudiantes de Administración y Dirección de Empresas. Universitas psychologica, 12 (3), 739-752.

Smink, J., y Schargel, F. P. (2013). Helping students graduate: A strategic approach to dropout prevention. Larchmont, NY: Eye on Education.

Smith, J. P. y Naylor, R. A. (2001). Dropping out of university: a statistical analysis of the probability of withdrawal for U.K. university students. Journal of Royal Statistical Society, 164(2), 389-405.

DOI: http://dx.doi.org/10.1111/1467-985X.00209

Tinto, V. (1975). Dropout from Higher Education: A theoretical Synthesis of Recent Research. Review of Educational Research, winter 1975, 45(1), 89-125. DOI: http://dx.doi.org/10.3102/00346543045001089

Trevizán, A. L., Beltrán, C. y Cosolito, P. (2009). Variables que condicionan la deserción y retención durante el trayecto universitario de 
alumnos de carrera de Ingeniería Agronómica de la Universidad Nacional de Rosario. Revista de Epistemología y Ciencias Humanas, 1, 85-95.

Vázquez, L. (2013). Los estudios de seguimiento de trayectorias escolares como estrategia de prevención del abandono de los estudios superiores. III Conferencia Latinoamericana Sobre el Abandono en la Educación Superior. Méjico, 13, 14 y 15 de noviembre de 2013.

Willcoxson, L. (2010). Factors affecting intention to leave in the first, second and third year of university studies: a semester-by-semester investigation. Higher Education Research \& Development, 29 (6), 623-639. DOI: http://dx.doi.org/10.1080/07294360.2010.501071

\section{Sobre los autores:}

\section{Ana Belén Bernardo Gutiérrez}

Profesora contratada doctora del Área de Psicología Evolutiva y de la Educación con dos quinquenios de docencia reconocidos. Destacada labor investigadora en varios proyectos nacionales e internacionales, así como en proyectos de innovación docente. Ha contribuido a la formación de personal investigador como directora de tesis doctorales y al desarrollo del conocimiento en el área de psicología evolutiva y de la educación como autora de numerosas publicaciones y comunicaciones científicas, trabajos gracias a los cuales ha sido acreditada como profesora titular de universidad por la ANECA en el año 2014.

Desde el año 2011 ha centrado su labor investigadora en el problema del abandono de los estudios de grado, destacando su participación en el Proyecto Integral de Orientación Académico-Profesional de la Universidad de Oviedo -financiado por el Ministerio de Educación, Cultura y Deporte- y en el Proyecto Alfa para la Gestión Universitaria Integral del Abandono -financiado por la Comisión Europea-.

Por otro lado, en el año 2012 comenzó su labor como Directora de Área de Colegios Mayores y Residencias Universitarias en el Vicerrectorado de Estudiantes. 


\section{Rebeca Cerezo Menéndez}

Profesora Ayudante Doctora del Departamento de Psicología, Universidad de Oviedo.

Doctora con una considerable trayectoria docente e investigadora, en parte gracias al carácter internacional de su formación, habiendo realizado estancias de investigación en universidades renombradas (ranking Shanghai) y participado en el desarrollo de tecnologías educativas para la mejora de resultados académicos en el nivel universitario. Destaca la labor realizada en el marco del Proyecto e-TRAL - Promoción de competencias de autorregulación del aprendizaje académico a través del campus virtual en educación superior (EDU2010-16231), desde el año 2010 hasta el 2013, que culmino en el desarrollo de la herramienta en formato online (patentada).

Su producción científica es muy amplia, tanto mediante la participación en congresos nacionales e internacionales y la elaboración de artículos publicados en revistas de alto impacto.

\section{José Carlos Núñez Pérez}

Catedrático de Universidad de Psicología Evolutiva y de la Educación en la Universidad de Oviedo. Actualmente es responsable del Programa de Doctorado de este departamento e imparte docencia en el Grado en Psicología y en Másteres de diferentes Universidades de España y Portugal. Dentro de sus líneas de investigación destaca el estudio científico de las dimensiones cognitivo-motivacional, emocional y social en los estudiantes con dificultades de aprendizaje, así como el análisis de estrategias de aprendizaje autorregulado.

Como investigador principal en multitud de proyectos de ámbito regional, nacional e internacional y destacado autor de textos científicos en diversos formatos, ha contribuido notablemente al desarrollo del conocimiento en éste área.

Actualmente desarrolla los cargos de Director del Departamento de Psicología, miembro del Consejo de Gobierno de la Universidad de Oviedo y Vocal de Dirección del Colegio Oficial de Psicólogos del Principado de Asturias. 


\section{Ellian Tuero Herrero}

Profesora Ayudante Doctora del Departamento de Psicología de la Universidad de Oviedo con una prometedora trayectoria docente e investigadora.

Como miembro del grupo de investigación de Aprendizaje Escolar, Dificultades y Rendimiento Académico ha participado en numerosas investigaciones que han dado lugar a la publicación de destacados trabajos académicos sobre dificultades y autorregulación del aprendizaje entre otros temas. Su contribución al desarrollo del conocimiento no se limita a la producción de estos trabajos, sino que la autora también destaca por su dedicación a la comunidad científica como revisora de renombradas revistas científicas y su participación en el comité científico de congresos nacionales e internacionales. Actualmente imparte docencia en los grados de psicología y magisterio.

\section{María Esteban García}

Licenciada en Pedagogía con Máster en e-Learning y redes sociales, con amplia experiencia profesional como coordinadora de programas educativos para la mejora de los resultados escolares en Psicólogos Sin Fronteras ONGD Ha participado como becaria de investigación en diversos proyectos, destacando el proyecto europeo Gestión Universitaria Integral del Abandono (GUIA), coordinado por la Universidad Politécnica de Madrid e implementado en veintiuna instituciones de educación superior. Actualmente se encuentra realizando el doctorado en Psicología y Educación bajo la dirección de José Carlos Núñez Pérez (catedrático de psicología) y Ana Belén Bernardo Gutiérrez (profesora contratada doctora).

\section{Para citar este artículo:}

Bernardo, A. et al. (2015). Predicción del abandono universitario: variables explicativas y medidas de prevención. Revista Fuentes, 16, Junio, pp. 63-84. [Fecha de consulta: dd/mm/aaaa].

http://www.revistafuentes.es/

D.O.I.: http://dx.doi.org/10.12795/revistafuentes.2015.i16.03 
Predicción del abandono universitario: variables explicativas y medidas de prevención 